\title{
评 述高温高密核物质形态研究专题
}

\section{质子自旋结构与RHIC上海夸克自旋分布的测量}

\author{
徐庆华 ${ }^{*}$, 梁作堂 ${ }^{*}$ \\ 山东大学前沿交叉科学青岛研究院, 粒子物理与粒子辐照教育部重点实验室, 青岛 266237 \\ *联系人, 徐庆华, E-mail: xuqh@sdu.edu.cn; 梁作堂, E-mail: liang@sdu.edu.cn \\ 收稿日期: 2019-02-18; 接受日期: 2019-04-25; 网络出版日期: 2019-07-31
}

\begin{abstract}
摘要对核子内味道分离的夸克自旋分布的研究是核子自旋结构研究的重要前沿课题之一, 也是RHIC自旋物理 研究的重要核心课题. 通过对极化质子-质子反应中W玻色子自旋不对称的测量, RHIC首次对 $\bar{u}$ 和 $\bar{d}$ 海夸克的自旋 分布 $\Delta \bar{u}$ 和 $\Delta \bar{d}$ 给出了高精度限制, 发现了 $\Delta \bar{u}$ 在 $0.05<x<0.25$ 区间内是正向极化的, 并显示核子内海夸克自旋结构存 在同位旋不对称, 即 $\Delta \bar{u}>\Delta \bar{d}$, 首次清楚地揭示出自旋分布的同位旋不对称与非极化时符号相反. 同时, RHIC还通 过对极化质子-质子反应中 $\bar{\Lambda}$ 超子极化转移的测量, 对反奇异海夸克 $\bar{s}$ 的螺旋度分布 $\Delta \bar{s}$ 以及横向极化度 $\delta \bar{s}$ 进行了 研究.
\end{abstract}

关键词 $W$ 自旋不对称, 海夸克自旋分布, 同位旋不对称, 超子极化

PACS: $24.85 .+\mathrm{p}, 13.75 . \mathrm{Cs}, 14.20 . \mathrm{Dh}, 13.85 . \mathrm{Ni}, 13.88 .+\mathrm{e}$

\section{1 引言}

核子结构是当前人类对物质结构认识的最深层 次, 一直是粒子物理与核物理活跃的前沿领域之一. 自 20 世纪60年代起，人们开始利用轻子核子深度非弹性 散射(DIS)实验研究核子结构. 由于轻子不参与强相互 作用, 是研究核子结构理想的探针. DIS实验被称作当 代卢瑟福实验，是研究核子结构非常有效的手段. DIS 实验的一系列重要发现，促使理论上建立起核子结构 的部分子模型 ${ }^{[1]}$, 成为目前普遍接受的核子结构图像 (如见最近简短综述[2]). 在此图像下, 参与高能反应的 核子由一束部分子构成，高能反应首先是这些部分子 之间的相互作用，部分子在核子中的动量分布由部分
子分布函数描述.

实验技术的进步，使人们对核子结构的研究逐渐 深入到自旋自由度. 在DIS实验中, 把初态轻子与核子 极化，人们可以测量所谓的自旋依赖的结构函 数 $g_{1}(x, Q)$. 在部分子模型中, $g_{1}(x, Q)$ 可以表示成自旋 依赖的部分子分布函数的线性叠加, 即

$$
g_{1}(x, Q)=\frac{1}{2} \sum_{i} \mathrm{e}_{q_{i}}^{2}\left[\Delta q_{i}(x, Q)+\Delta \bar{q}_{i}(x, Q)\right],
$$

其中 $\Delta q_{i}(x, Q)=q_{i}^{+}(x, Q)-q_{i}^{-}(x, Q)$ 是质子中部分子 $q_{i}$ 的螺旋度(Helicity), 即纵向自旋分布函数, 上标 + 或 表示部分子的螺旋度与质子自旋方向相同或相反; $x$ 是部分子的动量分数, $Q^{2}=-q^{2}, q$ 是交换光子的四动

引用格式: 徐庆华, 梁作堂. 质子自旋结构与RHIC 上海夸克自旋分布的测量. 中国科学: 物理学 力学 天文学, 2019, 49: 102007 Xu Q H, Liang Z T. Nucleon spin structure and measurements on sea quark polarization at RHIC (in Chinese). Sci Sin-Phys Mech Astron, 2019, 49: 102007, doi: 10.1360/SSPMA-2019-0048 
量. 利用实验对 $g_{1}(x, Q)$ 的测量结果, 可以计算

$\Gamma_{1}(Q)=\int_{0}^{1} \mathrm{~d} x g_{1}(x, Q)=\frac{1}{2} \sum_{i} \mathrm{e}_{q_{i}}^{2}\left[\Delta Q_{i}+\Delta \bar{Q}_{i}\right]$,

其中 $\Delta Q_{i}=\int_{0}^{1} \mathrm{~d} x \Delta q_{i}(x, Q)$ 是味道为 $q_{i}$ 的夸克对质子自旋 的贡献.

极化的DIS实验首先于1980年代在美国斯坦福直 线加速器中心(Stanford Linear Accelerator Center, SLAC) 开展 ${ }^{[3,4]}$, 紧接着欧洲核子研究中心(European Organization for Nuclear Research, CERN)也进行类似 的实验 ${ }^{[5,6]}$. 短短几年的时间, 实验上就发现超出人们 预期的结果. 1988年, CERN的EMC合作组发表了他们 的实验结果 ${ }^{[6]}$, 根据他们的测量结果, 再考虑到理论上 对称性等的限制, 人们得到

$$
\begin{aligned}
\Delta \Sigma & =\Delta U+\Delta U+\Delta D+\Delta \bar{D}+\Delta S+\Delta S \\
& =0.006 \pm 0.058 \pm 0.117 .
\end{aligned}
$$

该结果表明, 实验误差范围内, 质子内夸克反夸克自旋 对质子自旋的贡献几乎是零 ${ }^{[6]}$, 这与夸克模型的预期 完全不同，从而引发了所谓的“质子自旋危机”，也极 大地吸引了粒子物理与核物理学界的普遍兴趣，使自 旋结构成为核子结构研究最热门的课题之一.

EMC实验引发“质子自旋危机”以来，国际上各大 高能物理实验室都投入巨大力量开展该方向的测 量 $^{[5,7]}$. 开展的轻子核子深度非弹性散射实验包括 CERN的SMC 和COMPASS、德国汉堡同步加速器中 心(Deutsches Elektronen Synchrotron, DESY)的HERA 上的HERMES和美国杰佛逊国家实验室(Jefferson $\mathrm{Lab} ， \mathrm{JLab})$ 的CEBAF，他们分别采用极化的 $\mu^{+}$束、 $e^{+}$

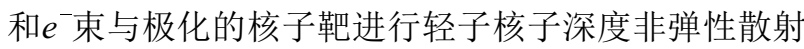
实验, 开展对 $g_{1}(x, Q)$ 的测量, 这些实验覆盖了不同的 $x$ 和 $Q$ 的区域, 获取互补的实验数据, 研究核子内部分 子的自旋分布.

随着理论与实验研究的进展, 人们很快认识到对 不同味道的夸克自旋分布研究的重要性以及单举的 DIS 实验的局限性. 上述单举 DIS 实验(即 $e N \rightarrow e X$ 和 $\mu N \rightarrow \mu X)$ 中轻子与核子的相互作用以电磁相互作 用为主导，在这些实验中人们能够测量的是(1)式给出 的 $g_{1}(x, Q)$, 它是以电荷平方为权重的不同味道夸克贡 献之和, 且不能区分夸克与反夸克, 即不能直接进行味 道分离的测量, 不能直接测量不同味道的夸克、反夸
克对自旋的贡献，只能依赖数据加理论模型分析间接 给出.

为了开展夸克味道分离的核子自旋分布测量，人 们开展了半单举DIS(如 $e^{-} N \rightarrow e^{-} h X$ ) 实验研究, 即通 过在末态粒子中探测一个强子 $h$ (如 $\pi$ 介子、 $K$ 介子等), 从而调节不同味道的夸克贡献的权重，达到味道分离 测量的目的. 这类实验迅速取得良好的进展 ${ }^{[5]}$, 对夸克 味道分离给出进一步的限制, 但同时得到尖锐的批评: 实验对夸克自旋分布味道分离的结论强烈地依赖于人 们对强子产生的描述即碎裂函数的精度, 大大增加了 测量结果的不确定度, 也使人们对核子内夸克自旋结 构味道分离的研究进展缓慢. 即使考虑各类DIS实验 数据的整体拟合(Global Fit)也只能给出海夸克自旋分 布比较粗粘的结果(图1). 更进一步的信息, 可参见近 期一篇很好的综述性论文 ${ }^{[8]}$.

在深入开展DIS实验的同时, 人们也认识到, 由于

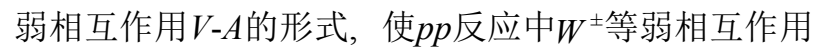

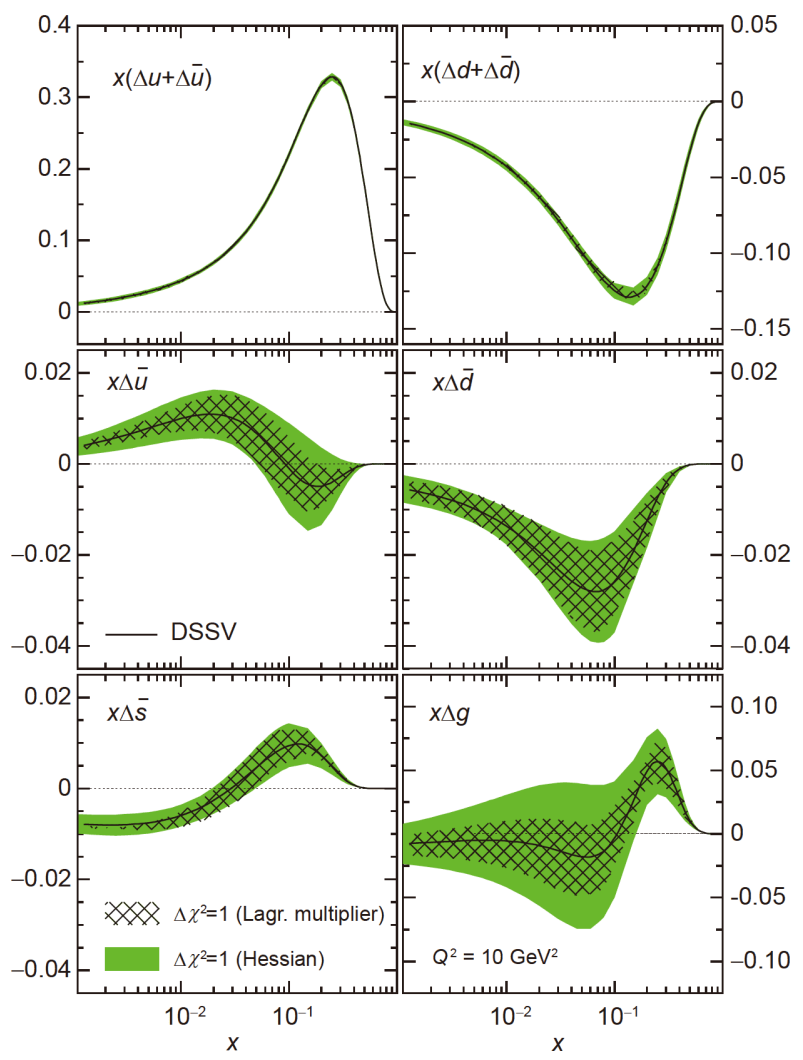

图 1 (网络版彩图)DSSV组整体参数化给出的极化部分子 分布函数. 该图取自文献[9]

Figure 1 (Color online) Helicity distribution functions for quarks and gluon in the proton from DSSV global analysis. Plot is from ref. [9]. 
中间玻色子的产生对夸克反夸克的螺旋度有自然的选 择，在极化的 $p p$ 反应中测量这些中间玻色子的产生可 以直接测量核子中相应味道的夸克的自旋分布. 并且, $W^{+}$和 $W^{-}$分别主要来源于 $u+\bar{d} \rightarrow W^{+}$和 $\bar{u}+d \rightarrow W^{-}$, 对它们的测量形成对夸克味道自然地分离, 是研究核 子自旋分布味道分离最理想的测量过程, 也成为RHIC 自旋物理规划设计的核心测量目标之一 ${ }^{[7]}$.

同时，理论分析还表明 ${ }^{[10,11]}$ ，在极化的 $p p$ 反应中， 测量末态超子和反超子的极化，特别是反超子极化的 测量，可以研究核子反奇异海夸克的自旋分布 $\Delta \bar{s}$, 在 横向极化情形下，还可研究横向自旋分布 $\delta \bar{s}$ ，为核子 内奇异海夸克的自旋分布的研究提供了一条重要的 途径.

至此，RHIC上STAR与PHENIX合作组不仅完成 了 $W^{ \pm}$自旋不对称的测量 ${ }^{[12-17]}$ ，还完成了 $\Lambda$ 和 $\Lambda$ 纵向和 横向极化转移的测量 ${ }^{[18-20]}$, 对核子内海夸克自旋分布 给出重要信息. 本文的目的就是总结RHIC对海夸克自 旋分布测量的重要结果，并给出对未来实验研究的 展望.

\section{$2 W$ 自旋不对称与海夸克自旋分布的同位 旋不对称}

在纵向极化的 $p p$ 碰撞中弱作用玻色子 $W$ 的自旋不 对称为海夸克自旋分布提供了一种非常干净的测量, 可以实现夸克螺旋度分布的味道分离，而不需要像半 单举DIS 实验过程中涉及强子碎裂函数. 因此是研究 质子自旋味道结构的一个强大而独特的工具，也是 RHIC自旋物理的核心物理目标之一 ${ }^{[7]}$.

\section{$2.1 W$ 自旋不对称与海夸克自旋分布}

在 $p p$ 碰撞中， $W$ 玻色子通过来自入射质子的夸克 和反夸克的湮灭产生. 在不考虑粲夸克的贡献时, $W^{+}$和 $W^{-}$分别主要来源于 $u+\bar{d} \rightarrow W^{+}$和 $\bar{u}+d \rightarrow W^{-}$这 两个过程. 并且, 由于标准模型中弱作用耦合是 $V-A$ 的 形式，参与反应产生 $W$ 玻色子的夸克和反夸克都是 $100 \%$ 纵向极化的，例如产生 $W^{+}$的 $u$ 必须是左手的即螺 旋度是负的，而 $\bar{d}$ 只能是右手的即螺旋度为正．图2 针 对 $W^{+}$产生给出了极化入射质子的螺旋度和来自该质 子的夸克反夸克螺旋度的组合情况. 可以看出, $W$ 产生
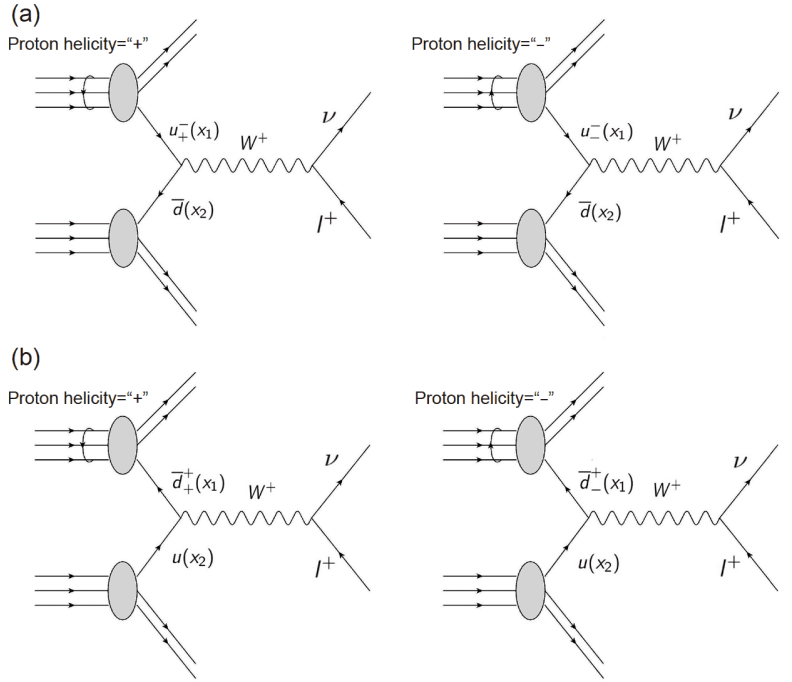

图 2 (a) 当 $u$ 夸克来自极化入射质子时 $W^{+}$玻色子通过 $u+\bar{d}$ 反应道的产生示意图; (b) 对应 $\bar{d}$ 夸克来自极化质子的示意图 Figure 2 (a) Diagram for $W^{+}$production via $u+\bar{d}$ channel with $u$ quark from polarized proton; (b) corresponding diagram with $\bar{d}$ antiquark from polarized proton.

截面依赖于入射质子的螺旋度和质子内夸克的自旋分 布, 从而导致如下宇称破坏的纵向单自旋不对称:

$A_{L}=\frac{\left(\sigma^{+}-\sigma^{-}\right)}{\left(\sigma^{+}+\sigma^{-}\right)}$

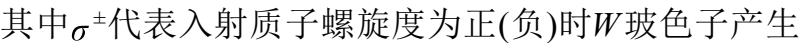
的微分截面.

在不考虑QCD高阶修正与粲夸克的贡献时， $W$ 自 旋不对称 $A_{L}$ 与部分子分布函数的关系为

$A_{L}^{W^{+}}\left(y_{w}\right)=\frac{-\Delta u\left(x_{1}\right) \bar{d}\left(x_{2}\right)+\Delta \bar{d}\left(x_{1}\right) u\left(x_{2}\right)}{u\left(x_{1}\right) \bar{d}\left(x_{2}\right)+\bar{d}\left(x_{1}\right) u\left(x_{2}\right)}$,

$A_{L}^{W^{-}}\left(y_{w}\right)=\frac{-\Delta d\left(x_{1}\right) \bar{u}\left(x_{2}\right)+\Delta \bar{u}\left(x_{1}\right) d\left(x_{2}\right)}{d\left(x_{1}\right) \bar{u}\left(x_{2}\right)+\bar{u}\left(x_{1}\right) d\left(x_{2}\right)}$,

其中 $\Delta u(x)$ 和 $\Delta \bar{d}(x)$ 分别表示上夸克和反下夸克的螺旋 度分布函数, $u(x)$ 和 $\bar{d}(x)$ 则是对应的非极化的分布函 数, $x_{1}$ 和 $x_{2}$ 分别表示来自极化的和非极化的入射质子 的夸克反夸克所携带的动量分数. 取极化质子的方向 为 $z$ 轴正方向， $x_{1}$ 和 $x_{2}$ 根据能动量守恒可以由 $W$ 玻色子 的质量 $m_{W}$ 和快度 $y_{W}$ 确定: $x_{1,2}=2 m_{W} \mathrm{e}^{ \pm y_{W}} / \sqrt{s}, s$ 为碰 撞质心系能量.

由(5)和(6)式可以清楚地看出, $W^{ \pm}$的产生主要包 含两部分贡献, 分对应图2中的(a)和(b)反应道. 第一部 
分对应产生 $W^{+}$的 $u$ 夸克来自于极化的质子, $\bar{d}$ 来自于非 极化的对撞质子；第二部分对应 $\bar{d}$ 来自于极化的质子, $u$ 夸克则来自于非极化的对撞质子. 由于价夸克主导 $x$ 比较大的区域，而海夸克主导小 $x$ 区域，这两部分贡献 分别主导 $y_{W}$ 为正且较大的区域和 $y_{W}$ 为负且绝对值较 大的区域，即分别主导正的大快度区域和负的大快度 区域. 因此，在这两个区域内，即对于较大的 $y_{W}>0$, $W^{ \pm}$的自旋不对称 $A_{L}^{W^{ \pm}}$分别正比于

$A_{L}^{W^{+}}\left(y_{W}\right) \sim-\frac{\Delta u\left(x_{1}\right)}{u\left(x_{1}\right)}, y_{W} \gg 0$,

$A_{L}^{W^{-}}\left(y_{W}\right) \sim-\frac{\Delta d\left(x_{1}\right)}{d\left(x_{1}\right)}, y_{W} \gg 0$,

且 $x_{1} \sim x_{W} \gg x_{2} \sim m_{W}^{2} / s x_{1}, \quad x_{W}=2 m_{W} \operatorname{sh} y_{W} / \sqrt{s}$ 是 $W$ 的动 量分数. 对于 $y_{W}<0$ 且绝对值比较大的区域, $W^{ \pm}$的自 旋不对称 $A_{L}^{W^{ \pm}}$分别正比于

$A_{L}^{W^{+}}\left(-\left|y_{W}\right|\right) \sim \frac{\Delta \bar{d}\left(x_{1}\right)}{\bar{d}\left(x_{1}\right)}, y_{W} \ll 0$,

$A_{L}^{W^{-}}\left(-\left|y_{W}\right|\right) \sim \frac{\Delta \bar{u}\left(x_{1}\right)}{\bar{u}\left(x_{1}\right)}, y_{W} \ll 0$,

且此时 $x_{2} \sim-x_{W} \gg x_{1} \sim m_{W}^{2} / s x_{2}$. 因此实验上通过测 量 $W$ 玻色子的纵向自旋不对称可以自然实现了核子自 旋结构的味道分离，特别是可以干净测量轻味海夸克 极化分布.

\subsection{RHIC 上 $W$ 自旋不对称的实验结果}

位于美国布鲁克海文国家实验室的RHIC既是世 界上首台高能相对论重离子对撞机也是世界上首台极 化的 $p p$ 对撞机. 为实现 $W$ 玻色子自旋不对称的测量, RHIC专门规划设计了 $500 \mathrm{GeV}$ 质心系能量下纵向极化 的 $p p$ 对撞实验, 并2009年开始首次成功运行, 随后又在 2011, 2012和2013年先后运行, 收集了较高统计量的 $W$ 产生实验数据. RHIC的两个实验组PHENIX和STAR都 对这些数据进行了分析，获取了 $500 \mathrm{GeV}$ 下极化 $p p$ 反 应中的 $W$ 纵向单自旋不对称的实验结果 ${ }^{[12-17]}$.

$\mathrm{RHIC}$ 上对 $W$ 玻色子的探测通过轻子衰变道 如 $W^{ \pm} \rightarrow e^{ \pm}+v_{e}$ 进行, 从而避免了碎裂函数的影响. PHENIX和STAR两个实验组分别利用2009年首次 $500 \mathrm{GeV}$ 下 $p p$ 碰撞试运行获取的数据, 对 $W$ 玻色子的产
生截面与单自旋不对称进行了测量 ${ }^{[12,15,21]}$ 。产生截面 由非极化的分布函数确定，PHENIX和STAR两个实验 组在中心快度区对产生截面和自旋不对称的实验测量 结果, 都在理论预期范围内, 检验了部分子分布函数的 普适性以及 $W$ 玻色子探测方法.

利用2011和2012年获取的大统计量 $500 \mathrm{GeV}$ 下 $p$ 对撞数据, STAR实验组首次测量了 $W$ 玻色子自旋不对 称随轻子赝快度 $\eta_{l}$ 的变化, 得到的结果如图3所示, 图 中的曲线是根据DIS实验结果对部分子自旋分布整体 参数化后(图1)的理论预期结果. 实验给出的是 $W$ 玻色 子自旋不对称随轻子赝快度 $\eta$ 变化的结果. 显然, 轻子 赝快度 $\eta_{l}$ 与 $W$ 的快度 $y_{W}$ 并不相同, $\eta_{l}=y_{W}+\eta_{l}^{*}, \eta_{l}^{*}$ 是 $W$ 静止坐标系内轻子的赝快度. 目前的理论框架已经在 次领头阶给出了随轻子 $\eta_{1}$ 变化的自旋不对称计算结 果 $^{[22,23]}$, 可以和实验结果直接比较.

从图3中可以清楚地看到, $A_{L}^{W^{-}}$在 $0<\eta<1$ 区域内 实验与理论也符合较好, 这个区域 $A_{L}^{W^{-}}$主要由 $d$ 夸克自 旋分布 $\Delta d(x)$ 确定; 而在 $\eta<0$ 的区域， $A_{L}^{W^{-}}$主要由 $\bar{u}$ 海夸 克自旋分布 $\Delta \bar{u}(x)$ 确定，理论不确定度高，尽管实验误

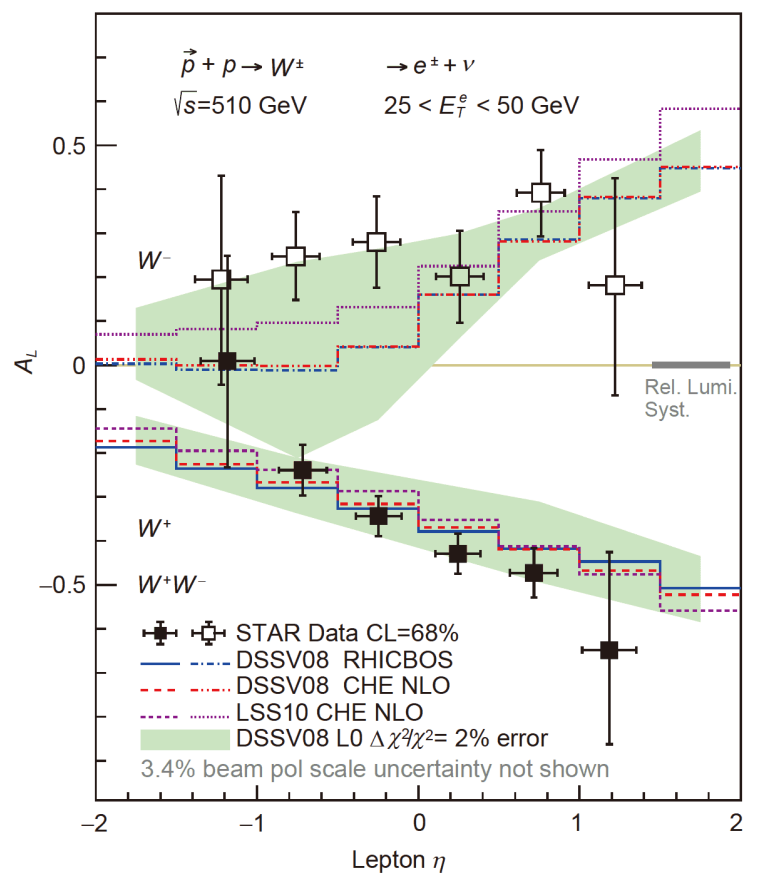

图 3 (网络版彩图)STAR实验组利用 $2011+2012$ 年实验数据 测得的 $W$ 坡色子的单自旋不对称结果. 该图取自文献[13]

Figure 3 (Color online) Results of single spin asymmetry for $W$ bosons from STAR 2011+2012 data sets. Plot is from ref. [13]. 
差也还很大，已可以看出与理论预期的偏离，表明 S TAR 的实验结果必定给出 $\Delta \bar{u}(x)$ 进一步的限制. 对 $A_{L}^{W^{+}}$而言, $\eta_{l}$ 与 $W$ 快度 $y_{W}$ 的关系不像 $A_{L}^{W^{-}}$那样分得清 楚 ${ }^{[23]}$, 导致整个 $\eta_{l}$ 区域内都由 $\Delta u(x)$ 主导, 而 $\Delta u(x)$ 在DIS 实验已得到较好确定，因而实验数据和理论预言比较 一致, $\bar{d}$ 夸克的贡献分布在整个 $\eta_{l}$ 区域, 高精度的 $A_{L}^{W^{+}}$ 数据可以给出 $\Delta \bar{d}(x)$ 的限制.

2013年RHIC专门为 $W$ 玻色子自旋实验运行了高 亮度的 $500 \mathrm{GeV}$ 极化 $p p$ 对撞, 获取了大统计量的 $W$ 产生 数据, 积分亮度为之前所有样本的总和的 3 倍左右. 利 用这些数据, STAR合作组迅速开展了 $W$ 玻色子自旋不 对称的测量, 得到的结果及其与之前利用2011+2012年 数据得到结果的比较见图 $4^{[14]}$. 图中也给出了 PHENIX 的测量结果，由于PHENIX探测器接收度小于STAR探 测器, PHENIX测量结果只覆盖 $|\eta|<0.5$ 的中心快度区 域 ${ }^{[16,17]}$. 可以看出, 这些测量结果是一致的.

图5给出了STAR实验组对2011-2013年所有数据 联合分析的测量结果以及与理论预期的比较. 这些理 论预期结果是基于相应的部分子分布函数参数化而得 到的，这些参数化是对已有实验数据进行整体拟合得

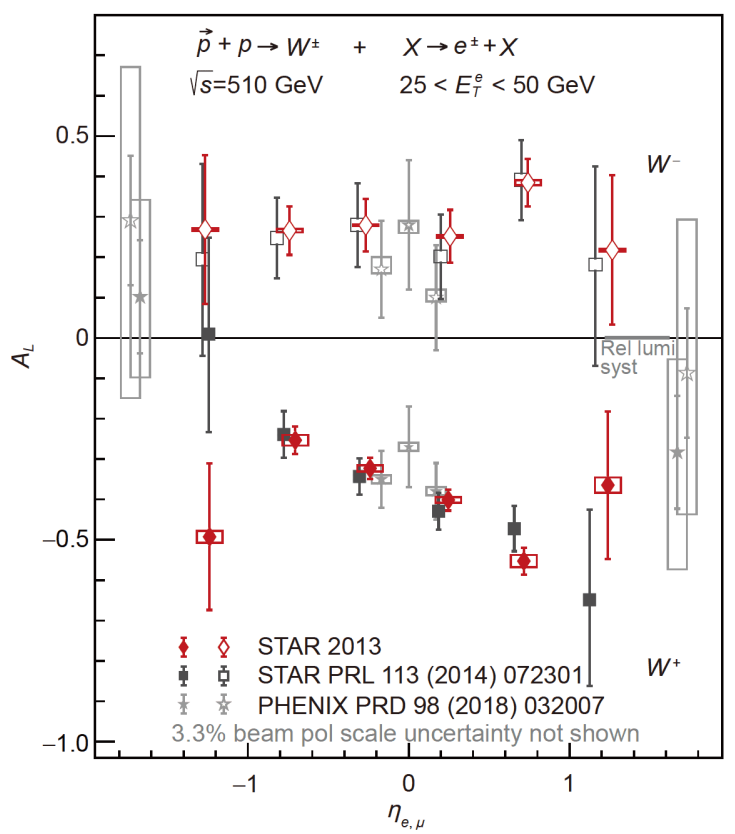

图 4 (网络版彩图)STAR实验组2013年实验数据的 $W$ 坡色 子自旋不对称的测量结果. 该图取自文献[14]

Figure 4 (Color online) Results of single spin asymmetry for $W$ bosons from STAR 2013 data set. Plot is from ref. [14].

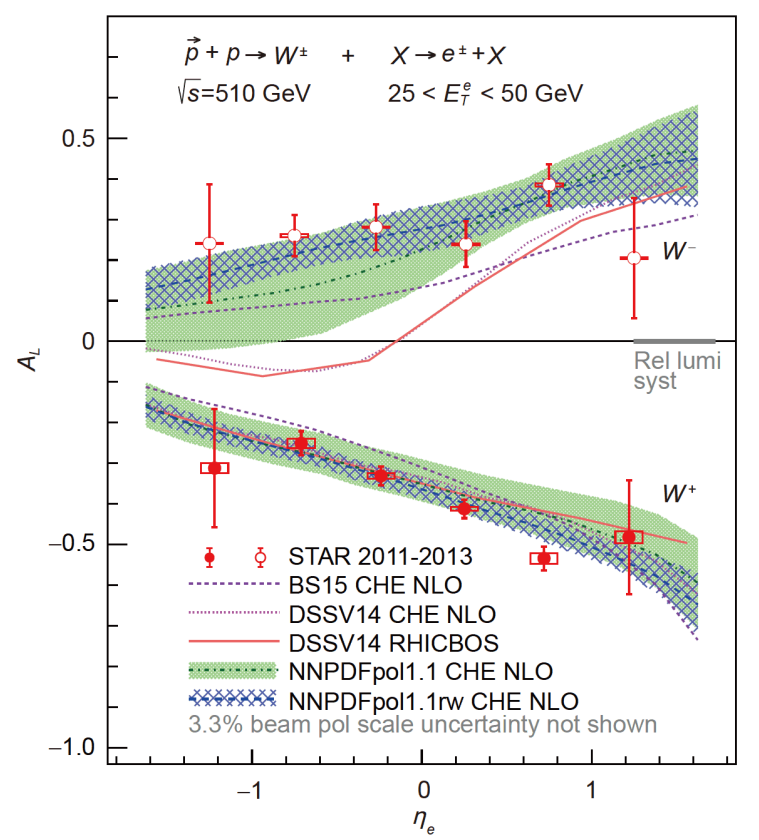

图 5 (网络版彩图)STAR实验组2011-2013年实验数据的 $W$ 玻色子自旋不对称的测量结果 ${ }^{[14]}$

Figure 5 (Color online) Results of single spin asymmetry for $W$ bosons from STAR 2011-2013 data set. Plot is from ref. [14].

到的，其中NNPDF pol1.1的参数化已考虑了STAR在 $2011+2012$ 年实验数据发表的 $W$ 玻色子自旋不对称数 据 ${ }^{[13]}$. 而NNPDF pol1.1rw是在NNPDF pol1.1的基础上 加入STAR实验2013年数据新结果后利用reweighting 重权重方法给出的 $A_{L}$ 结果，可以清晰地看到相应的不 确定度明显减小.

从图5所示 STAR实验数据结果可以清晰地看到, 不论是 $\eta_{e}>0$ 还是 $\eta_{e}<0$ 的区域, 都明显存在 $A_{L}^{W^{-}}>0$ 和 $A_{L}^{W^{+}}<0$ 的结论, 不仅验证了在价夸克主导 $x$ 比较大 的区域内 $\Delta u>0, \Delta d<0$ ，二者符号相反的结论(图1), 而且通过海夸克极化主导的 $\eta_{e}<0$ 的区域数据显 示 $\Delta \bar{u}>0, \Delta \bar{d}<0$, 即海夸克自旋分布也有明显的同位 旋对称破坏．这也是实验上首次给出海夸克自旋分布 同位旋对称破坏直接证据. 由这些数据得出的部分子 自旋分布的详细情况，则需要全面考虑已有各类实验 结果，进行部分子分布函数的整体拟合，相应结果在 下节给出.

\section{3 反海夸克自旋分布 $\Delta \bar{u}$ 与 $\Delta \bar{d}$ 不对称}

RHIC上 $W$ 坡色子纵向单自旋不对称测量结果发 
表后, 立即吸引了同行的重视. 国际上从事部分子极化 分布函数唯象学研究的几个主要的合作组都迅速把 RHIC数据考虑进来, 进行分布函数整体拟合 ${ }^{[24,25]}$. 图6 给出了NNPDFpol1.1关于海夸克自旋分布 $\Delta \bar{u}$ 与 $\Delta \bar{d}$ 在 考虑STAR实验2011+2012测量结果后拟合的结果 ${ }^{[24]}$ 以及与之前没有考虑STAR数据的DSSV参数化结果 ${ }^{[9]}$ 的对比. 可以看到, $\bar{u}$ 夸克的极化在 $x>0.1$ 的区域由负值 变为正值, 从而确定 $\bar{u}$ 夸克对核子自旋的贡献(即对 $x$ 积 分后)是正的. 对 $x$ 积分后的数值为 $\Delta \bar{u}=0.06 \pm 0.06$. 而
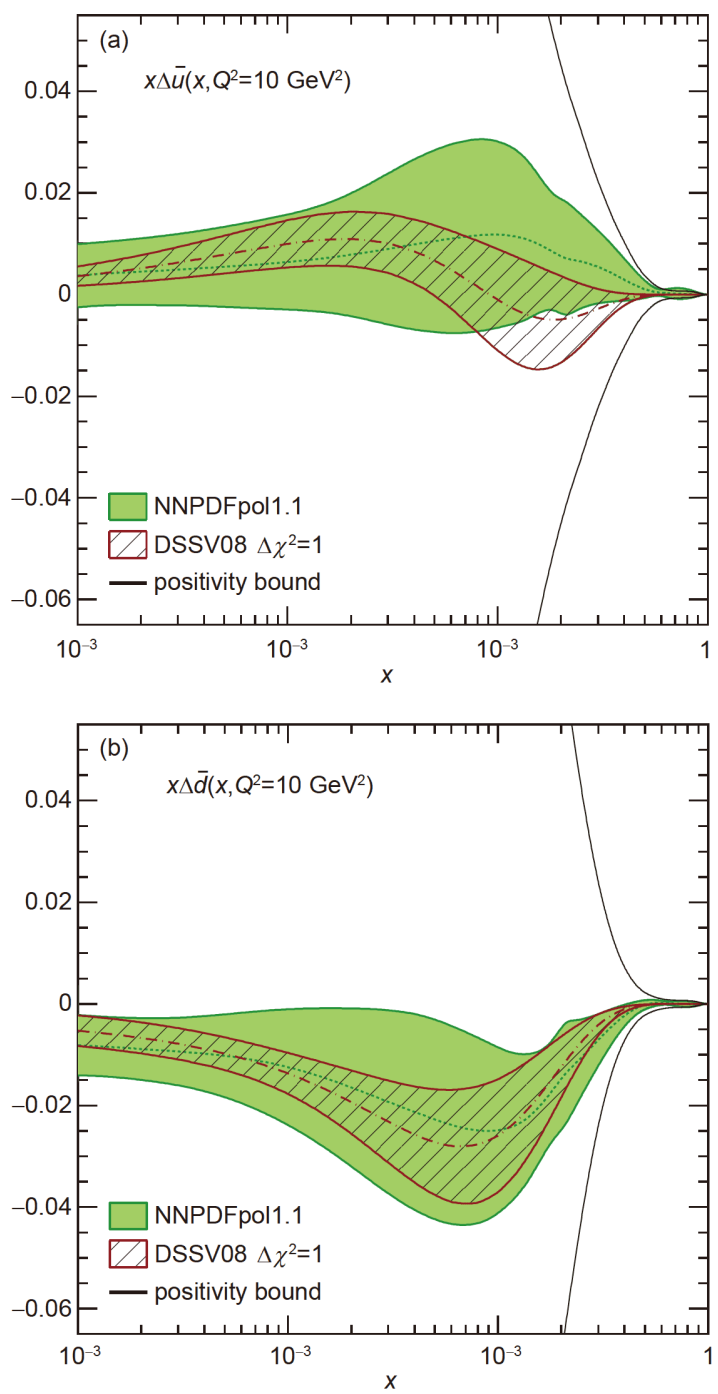

图 6 (网络版彩图)NNPDFpol1.1: $\bar{u}$ (a)和 $\bar{d}$ 夸克(b)的螺旋度 分布函数, 包括STAR实验组 $2011 \& 2012$ 实验结果的影响. 该 图取自文献[24]

Figure 6 (Color online) Helicity distribution functions for $\bar{u}$ (a) and $\bar{d}$ quark (b) from NNPDFpol1.1 global analysis, which included STAR $A_{L}$ results from $2011 \& 2012$ data sets. Plots are from ref. [24].
对 $\bar{d}$ 夸克，STAR实验组 $2011+2012$ 测量结果进一步 确定是负的，并显著缩小了不确定性， $\Delta \bar{d}=-0.11 \pm$ $0.06^{[24]}$. 图6中的阴影代表了参数化结果的误差，其中 NNPDFpol1.1给出 $1 \sigma$ 对应的不确定度, 而DSSV08是拟 合 $\Delta \chi^{2}=1$ 对应的不确定度 ${ }^{[9]}$.

图7给出了NNPDF参数化给出的海夸克 $\bar{u}$ 和 $\bar{d}$ 非极 化和纵向极化的分布函数的差异 ${ }^{[24,26]}$. 如果海夸克分 布存在同位旋 $S U(2)$ 对称，这个差别应该是 $0 . \mathrm{NNPDF}$ 参数化结果清晰地显示，海夸克分布的同位旋对称不 论是在非极化还是极化的情形下都是破坏的. 非极化 情形下海夸克分布的同位旋对称破坏，是非极化DIS 实验就确定的实验事实(参见近期的综述文章[8])，极 化情况下的同位旋破坏是RHIC上 $W$ 玻色子纵向单自 旋不对称实验结果的直接推论，并且通过对数据的参 数化，还清楚地看到，在目前实验数据比较敏感的区 域内, 极化与非极化情形下的同位旋破坏的符号是相反 的, 即非极化时 $\bar{u}(x)-\bar{d}(x)<0$, 极化时 $\Delta \bar{u}(x)-\Delta \bar{d}(x)>0$.

图8给出了在NNPDFpol1.1的基础上利用其提供 的reweighting方法, 加入STAR实验组2013年数据给出 的新结果后，极化海夸克分布味道对称破缺的新结 果 $^{[14]}$. 可以看到新结果的不确定性大幅降低，以更高 的显著性确认极化的部分子分布函数存在和非极化情 形相反的同位旋对称破缺.

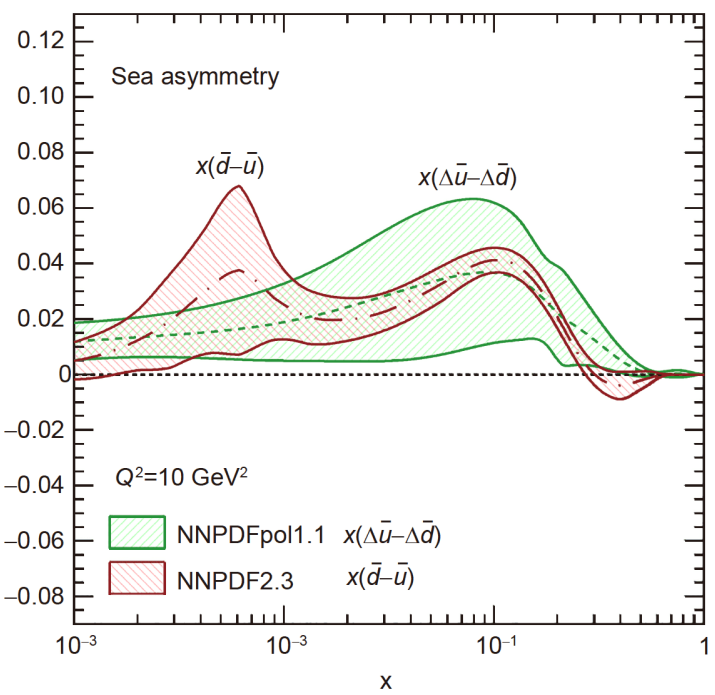

图 7 (网络版彩图)NNPDF给出的极化和非极化情形下海 夸克 $\bar{u}$ 和 $\bar{d}$ 分布函数的不对称. 该图取自文献[26]

Figure 7 (Color online) Sea quark asymmetry for polarized and unpolarized cases from NNPDF global fits. Plot is from ref. [26]. 


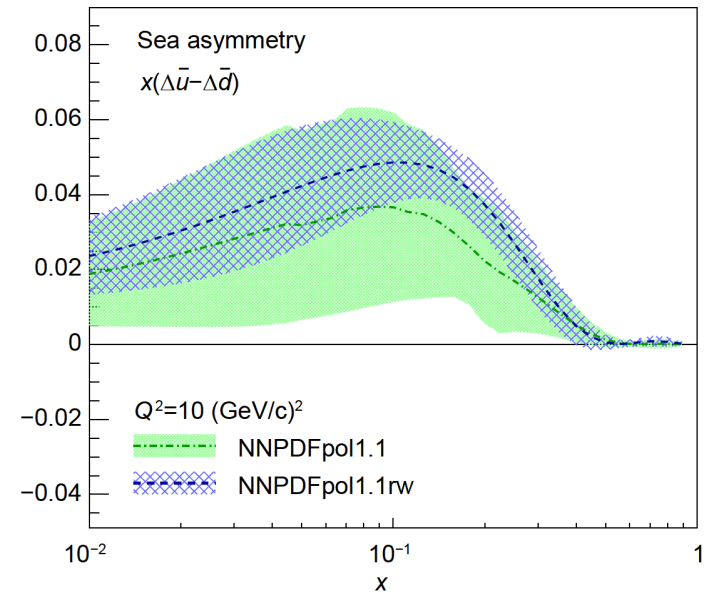

图 8 (网络版彩图)加入STAR 2013年数据后利用NNPDF给 出的 $\Delta \bar{u}, \Delta \bar{d}$ 不对称的结果以及与之前结果的对比. 该图取 自文献[14]

Figure 8 (Color online) Polarized sea asymmetry for $\Delta \bar{u}, \Delta \bar{d}$ from NNPDF reweighting after including STAR 2013 results, compared with previous results. Plot is from ref. [14].

\section{3 超子极化转移与奇异海夸克自旋分布}

海夸克自旋分布的测量中，更为困难的是奇异海 夸克的自旋分布 $\Delta s(x)$ 和 $\Delta \bar{s}(x)$. 与 $\Delta \bar{u}(x)$ 和 $\Delta \bar{d}(x)$ 相比, 目前与奇异海夸克自旋分布 $\Delta s(x)$ 和 $\Delta \bar{s}(x)$ 直接相关的 实验数据就更少. 在极化的 $p p$ 反应中测量末态超子的 极化有望为研究核子内奇异海夸克极化提供一条新的 途径.

\section{1 奇异海夸克自旋分布与超子极化的唯象学 分析}

我们知道, 超子都包含奇异价夸克, 特别是 $\Lambda$ 超子 包含一个奇异价夸克, 并且在简单夸克模型中, 这个奇 异夸克携带了整个 $\Lambda$ 超子的自旋. 因此, 极化的 $p p$ 反应 中，产生出的末态超子的极化势必在一定程度上携带 入射质子内奇异夸克极化的信息. 在因子化定理的框 架下，高能 $p p$ 反应中大横动量超子的产生截面可因子 化为部分子分布函数、碎裂函数及部分子散射截面三 部分的卷积. 相应地, 极化的超子产生截面就表示为极 化的部分子分布函数、极化碎裂函数和极化的硬散射 截面的卷积，其中部分子散射截面可由微扰QCD计算 给出. 因此, 在极化的 $p p$ 反应中测量超子的极化转移 应该可以给出极化部分子分布函数和极化碎裂函数的
信息. 当入射质子纵向极化时, 测量末态超子的纵向极 化可以研究奇异夸克螺旋度分布函数和纵向极化碎裂 函数; 而入射质子横向极化时, 通过测量超子的横向极 化来获取质子内夸克横向自旋分布函数和横向极化碎 裂函数的信息.

另一方面，超子弱衰变的性质使得超子的极化可 通过其弱衰变产物的角分布来测量, 为实验确定超子 极化提供了方便的方法, 也为利用超子极化研究初态 质子中夸克自旋分布与碎裂函数提供了可能.

文献 $[27,10]$ 较早注意到极化的 $p p$ 反应中 $\Lambda$ 超子及 反超子极化的这些特点, 采用唯象模型对末态超子极 化与初态质子内奇异夸克的自旋分布以及碎裂过程的 自旋依赖进行了分析, 而RHIC上STAR实验组完成了 超子纵向和横向自旋转移的测量 ${ }^{[18-20]}$. 以下简单总结 文献 $[27,28,10,11]$ 唯象分析以及STAR实验的测量 结果.

(1) 超子纵向自旋转移. 以 $\Lambda$ 超子为例, 在极化的 $p p$ 反应中, 产生的末态 $\Lambda$ 超子的纵向极化也称作纵向 自旋转移, 用 $D_{L L}$ 表示, 定义为

$D_{L L} \equiv \frac{\mathrm{d} \sigma_{p^{+} p \rightarrow \Lambda^{+} X}-\mathrm{d} \sigma_{p^{+} p \rightarrow \Lambda^{-} X}}{\mathrm{~d} \sigma_{p^{+} p \rightarrow \Lambda^{+} X}+\mathrm{d} \sigma_{p^{+} p \rightarrow \Lambda^{-} X}}=\frac{\mathrm{d} \Delta \sigma}{\mathrm{d} \sigma}$,

其中上标“+”和“-”表示粒子的纵向极化(螺旋度)正或 负; $\mathrm{d} \sigma$ 是微分截面, 在因子化定理框架下, 可以表示为 分布函数、碎裂函数与应散射界面的卷积，如极化的 微分截面就可表示为

$\frac{\mathrm{d} \Delta \sigma^{4}}{\mathrm{~d} p_{T}} \propto \int \mathrm{d} x_{a} \mathrm{~d} x_{b} \sum_{a b c d} \Delta f_{a}\left(x_{a}\right) f_{b}\left(x_{b}\right) \Delta D_{c}^{A} \mathrm{~d} \Delta \hat{\sigma}^{a b \rightarrow c d}$,

其中 $\Delta f_{a}\left(x_{a}\right)$ 是部分子的螺旋度分布函数, $\Delta D_{c}{ }^{A}(z)$ 是纵 向极化的碎裂函数; $\Delta \hat{\sigma}^{(a b \rightarrow c d)}$ 为极化的部分子散射截 面，这部分可以微扰计算得到; 其中的求和遍历所有 相关部分子散射过程，如 $q q \rightarrow q q, \bar{q} q \rightarrow \bar{q} q$, $q g \rightarrow q g, g g \rightarrow g g, g g \rightarrow \bar{q} q$ 等.

从(11)和(12)式可以清楚地看到, $p p$ 反应中到末 态 $\Lambda$ 超子的极化转移 $D_{L L}$ 与质子内极化分布函数和极 化的碎裂函数紧密相关, 但由于涉及众多子过程, 牵涉 因素较多, 能否给极化分布函数提供信息, 需要必要的 理论计算.

与极化的部分子分布函数相比，人们对各部分子 过程对各类超子产生截面的贡献有较多的理论与实验 
研究，这些都集成在一些高能反应Monte-Carlo事例产 生器中, 如PYTHIA ${ }^{[29]}$ 等. 利用这些事例产生器, 我们 可以给出比较可靠的结果供参考. 文献 $[27,10]$ 就采用 Monte-Carlo事例产生器PYTHIA对各个子过程的贡献 进行了系统分析，并采用不同模型对末态超子的极化 转移进行了计算, 图9给出的是对反超子的计算结果.

图9以及文献 $[10,11]$ 的分析表明, $p p$ 反应中大横动 量的反超子主要是由入射质子中反奇异夸克与其他部 分子发生硬散射后碎裂而产生的，特别是反 $\Lambda$ 超子的 极化对质子内反奇异夸克的自旋分布函数比较敏感, 利用不同的奇异夸克极化分布函数参数化的得到的反 $\Lambda$ 超子的极化结果差别明显, 因此反 $\Lambda$ 超子的极化转移 可以为反奇异夸克的自旋分布提供重要信息 ${ }^{[10]}$. 而其 他不同超子如 $\Sigma$ 和 $\Xi 及$ 其反粒子的极化，对不同味道海 夸克极化的依赖各不相同 ${ }^{[11]}$. 实验上对 $p p$ 反应中各类 超子与反超子极化的系统测量将为核子内奇异海夸克 自旋分布的研究提供一个新的方法.

(2) 超子横向自旋转移. 当入射质子横向极化时, 内部的夸克极化用横向极化分布函数(Transversity)来 描述，由于它是奇手征(Chiral-odd)的，不能在单举DIS 实验中测量, 目前几乎还没有直接的测量结果. 目前实 验上主要利用横向极化的半单举DIS实验中末态强子 的方位角不对称来抽取夸克横向极化分布函数的信 息，但这里它和另外一个奇手征的碎裂函数(称为 Collins函数)耦合在一起，因此对横向极化分布函数的提 取依赖于Collins函数的参数化.

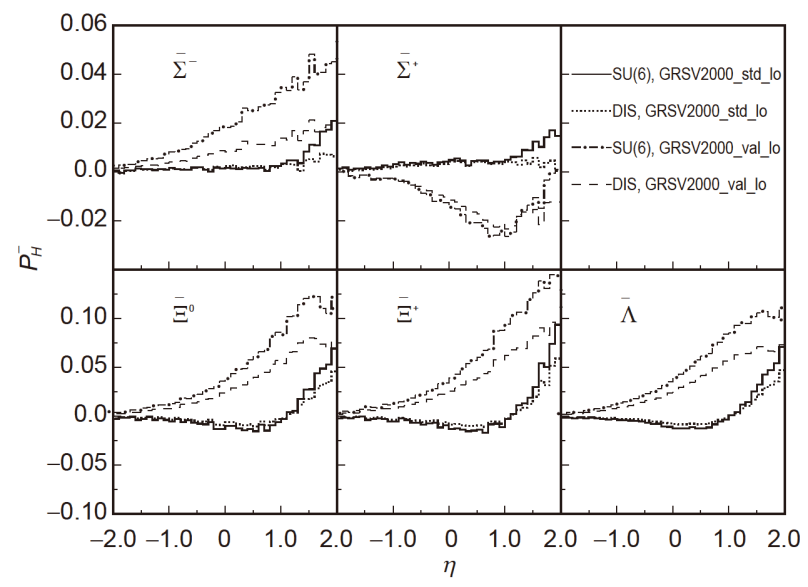

图 9 极化的 $p p$ 反应中反超子纵向自旋转移的理论模型计 算结果. 该图取自文献[11]

Figure 9 Results of longitudinal spin transfer for anti-hyperons in polarized $p p$ collisions. Plot is from ref. [11].
与上述纵向极化情形完全类似, 在横向极化的 $p p$ 反应中，末态超子与反超子的横向极化与夸克和反夸 克的横向极化分布函数关联在一起，因此测量超子的 横向极化转移可以给出横向极化分布函数的信息，在 横向极化的 $p p$ 反应中超子的横向极化也是少数几个可 以为横向极化分布函数提供信息的测量物理量之一 ${ }^{[10]}$.

对横向极化的 $p p$ 反应, $\Lambda$ 超子的横向极化转移 $D_{T T}$ 定义为

$$
\begin{aligned}
D_{T T} & \equiv \frac{\mathrm{d} \sigma(\hat{p} p \rightarrow \Lambda \hat{\Lambda} X)-\mathrm{d} \sigma\left(p^{\uparrow} p \rightarrow \Lambda^{\downarrow} X\right)}{\mathrm{d} \sigma\left(\hat{p} p \rightarrow \Lambda^{\uparrow} X\right)+\mathrm{d} \sigma\left(p^{\uparrow} p \rightarrow \Lambda^{\downarrow} X\right)} \\
& =\frac{\mathrm{d} \Delta_{T} \sigma^{\Lambda}}{\mathrm{d} \sigma^{\Lambda}},
\end{aligned}
$$

其中 $\sigma$ 为产生截面, 上标 $\uparrow /$ 表示粒子的极化方向. 与纵 向极化情形类似, 在只考虑领头阶的情况下, 极化的微 分截面可以因子化为横向极化的部分子分布函数、硬 散射子过程散射截面以及横向极化的碎裂函数的卷 积, 即

$$
\frac{\mathrm{d} \Delta_{T} \sigma^{\Lambda}}{\mathrm{d} p_{T}} \propto \int \mathrm{d} x_{a} \mathrm{~d} x_{b} \sum_{a b c d} \delta f_{a}\left(x_{a}\right) f_{b}\left(x_{b}\right) \Delta_{T} D_{c}^{\Lambda} \mathrm{d} \Delta_{T} \sigma^{a b \rightarrow c d},
$$

其中 $\Delta_{T} D_{c}^{\Lambda}$ 是横向极化的部分子碎裂产生横向极化超 子的碎裂函数, $\Delta_{T} \hat{\sigma}^{(a b \rightarrow c d)}$ 是硬散射子过程 $a b \rightarrow c d$ 横 向极化的散射截面.

由 $\Delta_{T} \hat{\sigma}^{(a b \rightarrow c d)}$ 决定的横向极化部分子极化转移是 可以微扰计算的, 各子过程的贡献与纵向极化是完全 类似，因此可以做类似的唯象学估算. 这类估算在文 献[10]给出. 与纵向极化情形下完全类似, 分析结果也 表明, 末态超子横向极化与质子内夸克的横向极化有 比较敏感的依赖, 对各类超子极化的系统测量可以为 夸克和反夸克的横向极化分布提供重要信息. 鉴于目 前对部分子横向极化分布的测量更加困难, 对横向极 化 pp 反应过程中超子和反超子自旋转移的测量是更有 特殊科学意义的课题.

\section{2 超子纵向自旋转移测量结果}

RHIC对撞机上的PHENIX和STAR实验组都较早 就对 $p p$ 反应的 $\Lambda$ 及 $\Lambda$ 超子的纵向自旋转移进行了测 量 $^{[30,31]}$. STAR实验组利用 2005 年获取的实验数据, 完 成了RHIC 上首次 $\Lambda$ 超子的纵向自旋转移测量 ${ }^{[18]}$, 随后 
STAR又在2009年获取了更大统计量数据, 对纵向自旋 转移进行了更高精度的测量 ${ }^{[19]}$.

RHIC 上对 $\Lambda$ 超子的极化是通过它的弱衰变过 程 $\Lambda \rightarrow p \pi^{-}$的角分布来确定的. 在 $\Lambda$ 静止坐标系内, 这 个角分布由式(15)给出

$\frac{\mathrm{d} N}{\mathrm{~d} \cos \theta^{*}} \sim \frac{1}{2} A\left(1+\alpha P_{\Lambda} \cos \theta^{*}\right)$,

其中 $\alpha=0.642$ 是 $\Lambda \rightarrow p \pi^{-}$过程的弱衰变常数, $P_{\Lambda}$ 是其 极化度, $\theta^{*}$ 是 $\Lambda$ 极化方向与 $\Lambda$ 静止系质子 $p$ 运动方向的 夹角, $A$ 是与探测器相关的接收度函数, 通常由模拟数 据得到, 显然 $A$ 将是系统误差的一个重要来源. STAR 实验组在利用 2003 年获取的数据进行测量时就指 出 $^{[30]}$, 在RHIC环境下, 可以利用超子产生的自旋不对 称来抽取超子的纵向极化转移, 即

$D_{L L}=\frac{1}{\alpha P_{\mathrm{b}}\left\langle\cos \theta^{*}\right\rangle} \frac{N^{+}-N^{-}}{N^{+}+N^{-}}$,

其中 $P_{\mathrm{b}}$ 是极化质子束的极化度, $N^{+}$和 $N^{-}$分别是质子的 极化是 + 或 - 时, 在一个小的 $\cos \theta *$ 区间内测到的 $\Lambda$ 的数 目, $\left\langle\cos \theta^{*}\right\rangle$ 是在这个小区间内的平均值. 这样的测量 方法不必依赖探测器接收度及效率，可以有效地降低 测量的系统误差. STAR首次采用这个方法, 给出纵向 极化 $p p$ 反应中 $\Lambda$ 与 $\bar{\Lambda}$ 的极化转移的测量结果 ${ }^{[18]}$. 这一 方法已被RHIC上普遍采用.

进一步利用2009年获取的更大统计量 $p p$ 数据, $\mathrm{STAR}$ 实验得到 $\Lambda$ 与 $\bar{\Lambda}$ 超子纵向自旋转移随横动量变化 的测量结果如图 10 所示 ${ }^{[19]}$. 在前向快度区 $\eta \sim 0.5$, 横

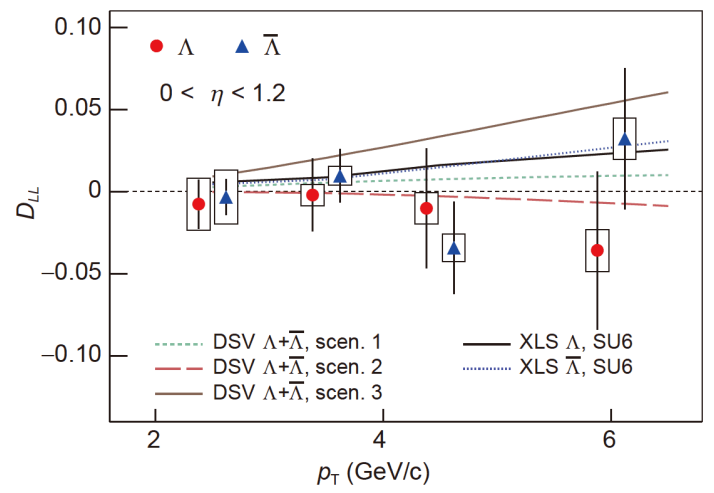

图 10 (网络版彩图)STAR实验组利用2009年数据测量超子 纵向自旋转移的实验结果. 图片取自文献[19]

Figure 10 (Color online) Experimental results of longitudinal spin transfer for hyperons from STAR experiment using 2009 data. Plot is from ref. [19].
动量为 $5.9 \mathrm{GeV}$ 时, $\Lambda$ 超子的 $D_{L L}=-0.036 \pm 0.048$ (stat) \pm 0.013 (sys); 对应 $\bar{\Lambda}$ 超子 $D_{L L}=0.032 \pm 0.043$ (stat) \pm 0.013 (sys).

图10还给出了不同理论预期结果. 其中DSV计算 的是超子和反超子合并在一起的结果 ${ }^{[32]}$, 三条不同曲 线代表了三个非常极端的极化碎裂函数的假设，图像 3 是假定不同味道的夸克对超子自旋贡献相同，基本 可以被数据排除. 由于目前测量的统计性在大横动量 区还较差，并且都基本是在中心快度区，其纵动量分 数很小 $\left(x_{F}<0.1\right)$, 这个区域对应的奇异海夸克与反夸 克的极化也比较小, 在目前的测量精度内还无法区分 $\Lambda$ 与 $\bar{\Lambda}$ 的自旋转移是否有差别.

\section{3 超子横向自旋转移测量结果}

与测量纵向极化不同, 在 $p p$ 反应中测量末态超子 的横向极化需要先确定极化的方向 ${ }^{[33,20]}$. 实验上有如 下三种可能测量方式:

(1) 以理论上给出的碎裂前部分子的极化方向作 为超子极化方向的横向极化转移, 即 (13) 式所定 义的 $D_{T T}$.

(2) 将超子极化方向选择在产生面(即超子与入射 质子运动方向构成的平面) 内的横向极化转移, 记作 $D_{S S}$.

（3）将超子极化方向选取为超子产生面的法线方 向, 记作 $D_{N N}$.

微扰QCD计算发现横向极化的部分子散射前后, 其横向极化方向相对散射面发生转动 ${ }^{[34]}, D_{T T}$ 测量了 末态超子沿出射夸克横向极化方向的自旋转移. 因此 上面后两种测量 $D_{N N}, D_{S S}$ 都是 $D_{T T}$ 的不同分量投影值. 在RHIC之前E704实验于1997年利用打靶实验测量过 $\Lambda$ 超子在产生面法线方向的横向自旋转移 $D_{N N}$, 在大动 量分数区域发现明显的横向自旋转移 ${ }^{[35]}$.

RHIC在2012年运行了横向极化的质心系能量为 $200 \mathrm{GeV}$ 的高能 $p p$ 对撞. STAR利用获取的实验数据完 成了 RHIC对 $\Lambda$ 超子及反超子的横向自旋转移 $D_{T T}$ 的首 次测量 ${ }^{[20]}$, 结果如图11所示.

为完成 $D_{T T}$ 测量, 首先需要确定碎裂前部分子的横 向极化方向, 因此需要考虑与喷注(Jet)的关联产生, 通 过喷注确定入射夸克横向极化方向的转动角度，进而 确定末态超子的横向极化方向 ${ }^{[34,20]}$. 


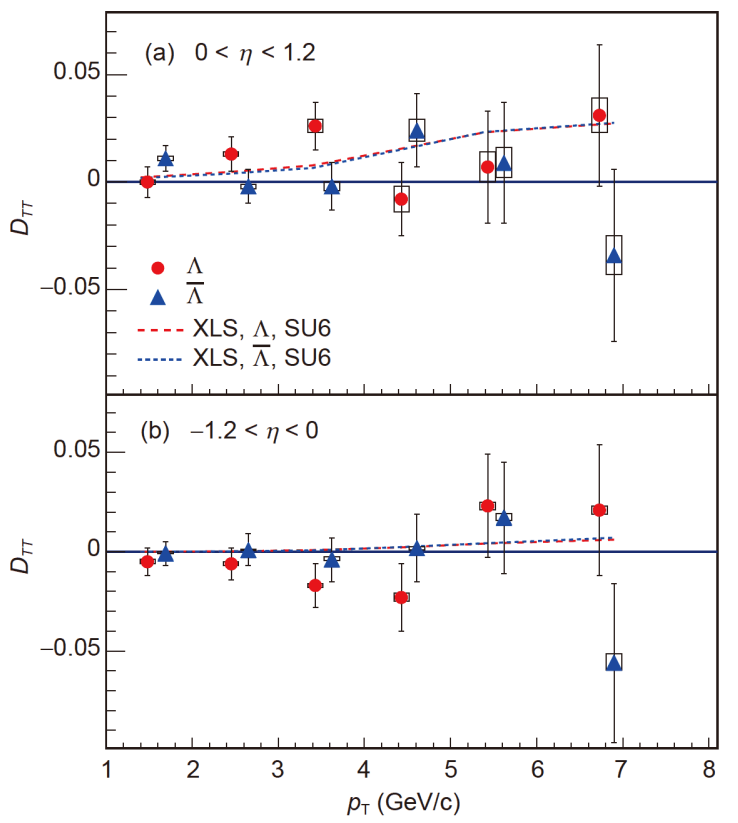

图 11 (网络版彩图)STAR实验组超子横向自旋转移的测量 结果. 该图取自文献[20]

Figure 11 (Color online) Experimental results on transverse spin transfer for hyperons from STAR experiment. Plot is from ref. [20].

STAR实验组对 $D_{T T}$ 测量中还首次利用交叉对称 (Cross-ratio)方法以消除测量中对相对亮度和接受度 的依赖 ${ }^{[20]}$, 即采用

$D_{T T}=\frac{1}{\alpha P_{\text {beam }}\left\langle\cos \theta^{*}\right\rangle} \frac{\sqrt{N_{+}^{\uparrow} N_{\unrhd}}-\sqrt{N_{+}^{\downarrow} N_{\perp}}}{\sqrt{N_{+}^{\uparrow} N_{\unrhd}}+\sqrt{N_{\ddagger}^{\downarrow} N_{-}}}$

来确定 $D_{T T}$. 这里的 $N_{+}^{\uparrow}$ 表示质子束极化为正时某 $\cos \theta^{*}$ 区间内的超子数目，而 $N_{-}^{\uparrow}$ 则表示质子束极化为正时相 应的 $-\cos \theta *$ 区间内的超子数目, 其余类推. 这里 $\theta^{*}$ 角由 前面确定的超子横向极化方向与 $\Lambda$ 静止系质子 $p$ 运动 方向的夹角给出. 因为(17)式不出现相对亮度和探测 器接收度, 由此大幅降低了测量的系统误差.

图11是STAR实验组在 $200 \mathrm{GeV}$ 的质子-质子碰撞 中分别在前向和后向赝快度区测量到的 $\Lambda$ 超子和反超 子横向自旋转移的实验结果 ${ }^{[20]}$. 结果随着超子横动量 的变化并不明显. 尽管在低横动量区达到了很高的测
量精度 $(<1 \%)$ ，测量结果在误差范围内没有观测到明 显的横向自旋转移. 在最高横动量 $7 \mathrm{GeV}$ 左右, 前向中 心快度区测量到的 $\Lambda$ 超子横向自旋转移为 $D_{T T}=0.031$ \pm 0.033 (stat) \pm 0.008 (sys); 反 $\Lambda$ 超子 $D_{T T}=-0.034 \pm 0.040$ (stat) \pm 0.009 (sys). 图中的理论计算是假定夸克横向极 化分布与纵向极化相同且奇异与反奇异夸克也相同时 得到的, 横向极化的碎裂函数用了价夸克模型即 $S U(6)$ 图像 ${ }^{[10]}$. 模型计算的结果反应出, 在目前实验测量覆 盖的运动学区域, 横向自旋转移的数值可能确实较小. 目前深度非弹散射实验的数据只对价夸克的横向自旋 分布给出了一些约束，而奇异夸克的信息仍然非常贵 $\Sigma^{[36-38]}$. 但如前所述, $\Lambda$ 超子独特的夸克结构和极化测 量途径, 使得超子自旋转移成为能够测量奇异夸克横 向自旋分布的桥梁.

\section{4 总结与展望}

世界上第一台极化的高能 $p p$ 对撞机RHIC的运行 为核子海夸克自旋分布的研究提供了很好的实验条 件. 特别是通过 $W$ 玻色子宇称破坏的单自旋不对称的 测量，取得了关于海夸克自旋分布的重要信息，首次 清楚地揭示出核子内海夸克自旋结构存在同位旋不对 称, 即 $\Delta \bar{u}>\Delta \bar{d}$, 且与非极化时符号相反.

对于奇异海夸克的自旋分布, 实验测量更加缺乏. 唯象理论模型研究表明, $p p$ 反应中超子的极化转移可 以为研究核子内奇异夸克极化提供一个新的途径. RHIC STAR实验改进了测量方法, 并完成了极化pp反 应中 $\bar{\Lambda}$ 超子纵向和横向极化转移的首次实验测量, 对 反奇异海夸克的螺旋度分布以及横向极化分布函数进 行了研究.

核子自旋结构的研究一直是强相互作用研究的前 沿课题之一, 海夸克的自旋分布是完全理解核子自旋 结构的重要内容之一. 目前计划中的STAR前向探测 器升级将RHIC的相关自旋测量推进到前向快度区, COMPASS及费米实验室上的极化Drell-Yan实验以及 未来EIC对撞机上高亮度的极化DIS实验都将为该方 向的研究提供很好的实验平台.

\section{参考文献}

1 Feynman R P. Photon-hadron Interactions. Boca Raton: CRC Press, 1972; Bjorken J D, Paschos E A. Inelastic electron-proton and $\gamma$-proton 
scattering and the structure of the nucleon. Phys Rev, 1969, 185: 1975-1982

2 Song Y K, Zhou J, Liang Z T. What is the structure and spin decomposition of the proton (in Chinese)? Chin Sci Bull, 2018, 63: 2546-2556 [宋玉 坤, 周剑, 梁作堂, 质子结构与质子自旋组成是什么? 科学通报, 2018, 63: 2546-2556]

3 Alguard M J, Ash W W, Baum G, et al. Deep inelastic scattering of polarized electrons by polarized protons. Phys Rev Lett, 1976, 37: 12611265; Deep-inelastic $e-p$ asymmetry measurements and comparison with the bjorken sum rule and models of proton spin structure. Phys Rev Lett, 1976, 41: 70-73

4 Baum G, et al. (E130 Collaboration). New measurement of deep-inelastic $e-p$ asymmetries. Phys Rev Lett, 1983, 51: 1135; Baum G, Bergström M R, Clendenin J E, et al. Measurement of asymmetry in spin-dependent $e-p$ resonance-region scattering. Phys Rev Lett, 1980, 45: 2000-2003

5 Aidala C A, Bass S D, Hasch D, et al. The spin structure of the nucleon. Rev Mod Phys, 2013, 85: 655-691, arXiv: 1209.2803

6 Ashman J, Badelek B, Baum G, et al. A measurement of the spin asymmetry and determination of the structure function g1 in deep inelastic muon-proton scattering. Phys Lett B, 1988, 206: 364-370; An investigation of the spin structure of the proton in deep inelastic scattering of polarised muons on polarised protons. Nucl Phys B, 1989, 328: 1-35

7 Bunce G, Naohito S, Soffer J, et al. Prospects for spin physics at RHIC. Annu Rev Nucl Part Sci, 2000, 50: 525-575

8 Chang W C, Peng J C. Flavor structure of the nucleon sea. Prog Particle Nucl Phys, 2014, 79: 95-135, arXiv: 1406.1260

9 de Florian D, Sassot R, Stratmann M, et al. Extraction of spin-dependent parton densities and their uncertainties. Phys Rev D, 2009, 80: 034030, arXiv: 0904.3821

10 Xu Q H, Liang Z T, Sichtermann E. Anti-lambda polarization in high energy pp collisions with polarized beams. Phys Rev D, 2006, 73: 077503

11 Chen Y, Liang Z T, Sichtermann E, et al. Antihyperon polarization in high energy pp collisions with polarized beams. Phys Rev D, 2008, 78: 054007, arXiv: 0707.0534

12 Aggarwal M M, Ahammed Z, Alakhverdyants A V, et al. Measurement of the parity-violating longitudinal single-spin asymmetry for $W^{ \pm}$boson production in polarized proton-proton collisions at $\sqrt{s}=500 \mathrm{GeV}$. Phys Rev Lett, 2011, 106: 062002, arXiv: 1009.0326

13 Adamczyk L, Adkins J K, Agakishiev G, et al. Measurement of longitudinal spin asymmetries for weak boson production in polarized protonproton collisions at RHIC. Phys Rev Lett, 2014, 113: 072301

14 Adam J, Adamczyk L, Adams J R, et al. Measurement of the longitudinal spin asymmetries for weak boson production in proton-proton collisions at $\sqrt{S}=510 \mathrm{GeV}$. Phys Rev D, 2019, 99: 051102, arXiv: 1812.04817

15 Adare A, Afanasiev S, Aidala C, et al. Cross section and parity-violating spin asymmetries of $W^{ \pm}$boson production in polarized $p+p$ collisions at $\sqrt{S}=500 \mathrm{GeV}$. Phys Rev Lett, 2011, 106: 062001, arXiv: 1009.0505

16 Adare A, Aidala C, Ajitanand N N, et al. Measurement of parity-violating spin asymmetries in $W^{ \pm}$production at midrapidity in longitudinally polarized $p+p$ collisions. Phys Rev D, 2016, 93: 051103, arXiv: 1504.07451

17 Adare A, Aidala C, Ajitanand N N, et al. Cross section and longitudinal single-spin asymmetry $A_{L}$ for forward $W^{ \pm} \rightarrow \mu^{ \pm} v$ production in polarized $p+p$ collisions at $\sqrt{s}=510 \mathrm{GeV}$. Phys Rev D, 2018, 98: 032007, arXiv: 1804.04181

18 Abelev B I, Aggarwal M M, Ahammed Z, et al. Longitudinal spin transfer to $\Lambda$ and $\bar{\Lambda}$ hyperons in polarized proton-proton collisions at $\sqrt{s}=$ 200 GeV. Phys Rev D, 2009, 80: 111102, arXiv: 0910.1428

19 Adam J, Adamczyk L, Adams J R, et al. Improved measurement of the longitudinal spin transfer to $\Lambda$ and $\bar{\Lambda}$ hyperons in polarized proton-proton collisions at $\sqrt{s}=200 \mathrm{GeV}$. Phys Rev D, 2018, 98: 112009, arXiv: 1808.07634

20 Adam J, Adamczyk L, Adams J R, et al. Transverse spin transfer to $\Lambda$ and $\bar{\Lambda}$ hyperons in polarized proton-proton collisions at $\sqrt{s}=200 \mathrm{GeV}$. Phys Rev D, 2018, 98: 091103

21 Adamczyk L, Agakishiev G, Aggarwal M M, et al. Measurement of the $W \rightarrow e v$ and $Z / \gamma^{*} \rightarrow e^{+} e^{-}$production cross sections at mid-rapidity in proton-proton collisions at $\sqrt{S}=500 \mathrm{GeV}$. Phys Rev D, 2012, 85: 092010, arXiv: 1112.2980

22 Nadolsky P M, Yuan C P. Single-spin physics with weak bosons at RHIC. Nucl Phys B, 2003, 666: 31-55

23 de Florian D, Vogelsang W. Helicity parton distributions from spin asymmetries in $W$-boson production at RHIC. Phys Rev D, 2010, 81: 094020, arXiv: 1003.4533

24 Nocera E R, Ball R D, Forte S, et al. A first unbiased global determination of polarized PDFs and their uncertainties. Nucl Phys B, 2014, 887: 276-308, arXiv: 1406.5539

25 de Florian D, Sassot R, Stratmann M, et al. Evidence for polarization of gluons in the proton. Phys Rev Lett, 2014, 113: 012001, arXiv: 1404.4293 
26 Nocera E R. Flavor asymmetry of the polarized nucleon sea. PoS, 2014, DIS2014: 204

$27 \mathrm{Xu}$ Q H, Liu C X, Liang Z T. Longitudinal polarization of hyperons in high $p_{\perp}$ jets in singly polarized $p p$ collisions at high energies. Phys Rev D, 2002, 65: 114008

$28 \mathrm{Xu}$ Q H, Liang Z T. Probing gluon helicity distribution and quark transversity through hyperon polarization in singly polarized $p p$ collisions. Phys Rev D, 2004, 70: 034015

29 Sjöstrand T, Mrenna S, Skands P. PYTHIA 6.4 physics and manual. J High Energy Phys, 2006, 605: 26

$30 \mathrm{Xu} \mathrm{Q}$, et al. (STAR Collaboration). Measurements of lambda and anti-lambda polarization in longitudinally polarized proton-proton collisions at 200-GeV at STAR. AIP Conf Proc, 2006, 842: 71-73

31 Han R, et al. (PHENIX Collaboration). The polarization of anti-Lambda in $\sqrt{s}=200-\mathrm{GeV}$ polarized proton-proton collision. AIP Conf Proc, 2007, 915: 432-435

32 de Florian D, Stratmann M, Vogelsang W. Polarized $\Lambda$-baryon production in $p p$ collisions. Phys Rev Lett, 1998, 81: 530-533

33 de Florian D, Soffer J, Stratmann M, et al. Bounds on transverse spin asymmetries for $\Lambda$ baryon production in pp collisions at BNL RHIC. Phys Lett B, 1998, 439: 176-182

34 Collins J C, Heppelmann S F, Ladinsky G A. Measuring transversity densities in singly polarized hadron-hadron and lepton-hadron collisions. Nucl Phys B, 1994, 420: 565-582

35 Bravar A, Adams D L, Akchurin N, et al. Spin transfer in inclusive $\Lambda^{0}$ production by transversely polarized protons at $200 \mathrm{GeV} / c$. Phys Rev Lett, 1997, 78: 4003-4006

36 Anselmino M, Boglione M, D'Alesio U, et al. Simultaneous extraction of transversity and collins functions from new semi-inclusive deep inelastic scattering and $e^{+} e^{-}$data. Phys Rev D, 2013, 87: 094019

37 Kang Z B, Prokudin A, Sun P, et al. Extraction of quark transversity distribution and collins fragmentation functions with QCD evolution. Phys Rev D, 2016, 93: 014009, arXiv: 1505.05589

38 Radici M, Bacchetta A. First extraction of transversity from a global analysis of electron-proton and proton-proton data. Phys Rev Lett, 2018, 120: 192001, arXiv: 1802.05212 


\title{
Nucleon spin structure and measurements on sea quark polarization at RHIC
}

\author{
XU Qing-Hua* \& LIANG Zuo-Tang* \\ Key Laboratory of Particle Physics and Particle Irradiation (MoE), Institute of Frontier and Interdisciplinary Science, \\ Shandong University, Qingdao 266237, China
}

\begin{abstract}
The nucleon spin structure in terms of its parton constituents is a fundamental question in QCD. The sea quark contribution to the nucleon spin is an important piece for a complete understanding of the nucleon spin structure. The production of $W$ bosons in longitudinally polarized proton-proton collisions at RHIC provides a unique probe for the sea quark polarization, through the parity-violating single-spin asymmetry $A_{L}=\left(\sigma^{+}-\sigma^{-}\right) /\left(\sigma^{+}+\sigma^{-}\right)$. At RHIC, $W$ 's can be detected via the leptonic channel $W \rightarrow e v$ with the kinematics of the decay lepton alone. Longitudinally polarized proton-proton collision events at $\sqrt{S}=500 \mathrm{GeV}$ were recorded at RHIC through the years 2009, 2011, 2012 and 2013. Both STAR and PHENIX experiments completed their measurements on longitudinal single spin asymmetries for $W$ bosons as a function of decay lepton rapidity. These results provide new constraints on sea quark polarizations through the QCD global analyses, which clearly indicate the existence of a flavor asymmetry in the polarization of the light-quark sea, $\Delta \bar{u}>\Delta \bar{d}$, in the proton for parton momentum fraction range of $0.05<x<0.25$. This is opposite to the flavor asymmetry observed in the unpolarized quark distributions, where $\bar{d}>\bar{u}$ over a wide $x$ range has been observed. On the other hand, the phenomenological studies showed that the spin transfer to $\bar{\Lambda}$ anti-hyperons provides a natural connection to helicity or transversity distributions of strange and anti-strange quarks in the proton. STAR experiment made the first and an improved measurement on the longitudinal spin transfer $D_{L L}$ to $\Lambda$ and $\bar{\Lambda}$ hyperons in longitudinally polarized proton-proton collisions at $200 \mathrm{GeV}$. STAR also completed the first measurement on transverse spin transfer $D_{L L}$ to $\Lambda$ and $\bar{\Lambda}$ hyperons in transversely polarized proton-proton collisions at $200 \mathrm{GeV}$. These data and future measurements with higher precision and larger momentum fraction in the forward region at RHIC will shed new lights on the polarized parton distributions for strange and anti-strange quark in the nucleon.
\end{abstract}

$W$ spin asymmetry, polarized parton distributions of sea quarks, iso-spin asymmetry, hyperon spin transfer

PACS: $24.85 .+\mathrm{p}, 13.75 . \mathrm{Cs}, 14.20 . \mathrm{Dh}, 13.85 . \mathrm{Ni}, 13.88 .+\mathrm{e}$

doi: $10.1360 /$ SSPMA-2019-0048 